\title{
Herramientas para desarrollar el reconocimiento ético en estudiantes universitarios: Una experiencia de aprendizaje con Pokémon Go
}

\author{
José Carlos Vázquez Parra ${ }^{1}$, Adriana Encinas Soto $^{2}$ y Ezequiel García Béjar ${ }^{3}$ \\ ${ }^{123}$ Departamento de Formación Humanística y Ciudadana \\ Campus Guadalajara Tecnológico de Monterrey
}

Recibido: 04 de noviembre de 2016

Aceptado: 01 de enero 2017

\section{Resumen}

El presente artículo, tiene por objetivo el exponer una experiencia de aprendizaje en un grupo de estudiantes universitarios de una institución privada de la ciudad de Guadalajara, México, mismo en el que por medio de un juego de realidad aumentada, Pokémon Go, se consiguió aportar a la sub-competencia ética de reconocimiento. Se considera que lo planteado es valioso, ya que contribuye al plantear un ejemplo en el que la formación ética se puede ayudar de las tendencias tecnológicas que resultan relevantes para los jóvenes usuarios digitales.

Palabras clave: Realidad aumentada, competencias, sentido humano, ética, educación.

\begin{abstract}
This article aims to expose an experience collaborative learning in a group of college students from a private institution in the city of Guadalajara, Mexico, one in which through an augmented reality game, Pokémon Go, it was possible to contribute to the ethical recognition. Although the limitation of this text is recognized, to only focus on this mobile application, it is considered that the issues raised is valuable in itself, as it contributes to raising an example that ethics training can help trends or issues time (trending topics) that are relevant for young digital users.
\end{abstract}

Keywords:?Augmented reality, skills, human sense, ethics, education.

${ }^{1}$ Correspondencia al autor

E-mail: jcvazquezp@itesm.mx 


\section{Introducción}

No se puede negar que desde la década de los noventa la humanidad, ha puesto a las instituciones educativas en una situación cada vez más complicada al momento de formar a los futuros profesionistas que serán los responsables del mundo moderno, todo esto como consecuencia de las cambiantes estructuras sociales, políticas, económicas y tecnológicas contemporáneas. Esto, ha ocasionado que los educadores pongan una mayor atención en la necesidad de desarrollar múltiples competencias en los estudiantes, ya que se han percatado de que no basta con un desarrollo disciplinar, cuando los aspectos sociales o personales no muestran un progreso consistente. Lo anterior ha contribuido a la clara oposición hacia los sistemas educativos de repetición, mismos que solo buscan la saturación del alumno con información técnica, lo que es considerado como un "sin sentido" en un mundo contemporáneo que cada vez exige más a los individuos (Fernández \& Velazco, 2003).

Por si esto fuera poco, la evolución de la concepción de la profesión, en la que ya no solo se percibe por su aportación a un trabajo, sino también por su contribución social, ha obligado a que las instituciones educativas desarrollen mapas curriculares mixtos, donde puedan dotar a sus estudiantes de herramientas adicionales, con las cuales éstos respondan a un mayor número de situaciones, sean de un ámbito laboral, social o personal (Toffer, 1992). Por ello, es que autores como Nieto (2000) consideran que es responsabilidad de las universidades no simplemente dotar al futuro profesionista de técnicas necesarias para ejercer su profesión, sino que también, se les debe nutrir de materiales que complementen la plenitud de su desarrollo formativo como personas, profesionistas y ciudadanos.

Una referencia de esta tendencia educativa es lo realizado por la Universidad de Harvard durante la administración de Derek Bok, la cual propuso la necesidad de fortalecer a sus estudiantes con competencias que no solo desarrollaran sus capacidades profesionales, sino también les permitieran enfrentar y resolver situaciones problemáticas que los afectarán en el terreno social y personal (Harvard, 2015). Así, se dispuso un programa de formación ética que no simplemente se basará en cursos aislados para los estudiantes, sino más bien, en una profunda modificación curricular que impactará la formación misma de los jóvenes, colocando a la ética 
como un eje rector de los programas profesionales de la institución (Iracheta, 2011).

Esta propuesta permitió la fundación de la Edmond J. Safra Foundation Center for Ethics, institución que actualmente se dedica no solo a la enseñanza y la investigación de la ética a nivel universitario, sino que también se enfoca al análisis y resolución de problemáticas éticas de la vida pública, el gobierno, los negocios, las empresas y los grupos de profesionistas en los Estados Unidos (Safra, 2015).

De manera complementaria a este antecedente, en 1990 la educadora francesa Francis Best publicó un artículo en el que, como tema sustancial, planteaba la necesidad de promover en los jóvenes estudiantes valores éticos que les permitieran reconocer y resolver situaciones dilemáticas de la sociedad contemporánea, pues consideraba que la expansión de las economías nacionales y el mundo cada vez más globalizado, requeriría de los futuros profesionistas competencias de análisis que fomentarán el desarrollo moral de los individuos (Best, 2015). Considerando la propuesta de Best, la UNESCO planteó que como parte sustancial de los proyectos educativos del siglo XXI, la inclusión de la formación ética en todos los niveles escolares resultaba necesaria, pues era primordial recuperar en los jóvenes un sentido humano y un verdadero conocimiento e interés por el desarrollo social de sus comunidades, en contraposición al bienestar individualista que se venía fomentando por las economías modernas (Iracheta, 2011). Así como la Universidad de Harvard, otras instituciones alrededor del mundo se enfocaron en la necesidad de la enseñanza de valores éticos en los estudiantes, impactando sus programas curriculares tanto a nivel transversal, como generando cursos específicos de formación humanística y reflexión ética.

El presente artículo, tiene por objetivo el exponer una experiencia de aprendizaje colaborativo en un grupo de estudiantes universitarios de una institución privada de la ciudad de Guadalajara, México, mismo en el que por medio de un juego de realidad aumentada para dispositivos móviles, Pokémon Go, se consiguió aportar a la sub-competencia ética de reconocimiento, demostrando que la formación con sentido humano puede gestarse de múltiples maneras, y no únicamente por medio de la reflexión teórica. Aunque se reconoce el limitante de este texto, al solo enfocarse en esta aplicación móvil, se considera que lo planteado es valioso por sí mismo, ya que contribuye al plantear un ejemplo en el que la formación ética se puede ayudar de las tendencias o temas de momento (trending topics) que resultan relevantes para los jóvenes usuarios digitales 


\section{Marco teórico}

\section{El uso de aplicaciones móviles como herramientas educativas}

Así como la sociedad está evolucionando a partir de la revolución digital, no es lógico que la educación no se viera impactada por este fenómeno. Por ende, los pedagogos y docentes se han visto en la labor de incorporar las tecnologías de la información y la comunicación (TIC) como instrumentos de enseñanza, convirtiendo a éstas tecnologías en los mejores aliados para favorecer el desarrollo de competencias en los nuevos profesionistas (CEFyTIC, 2014).

Aunque en el ámbito teórico se han elevado muchas opiniones, a favor y en contra, acerca del uso de las TIC en la educación, es labor del docente estar a la vanguardia de los cambios del mundo y por ende, desligar la educación del uso de las TIC es algo que no debe permitirse (Hernández \& Muñoz, 2012). Investigaciones de Ruiz (2004) y Sancho (2006) han demostrado que las tecnologías aportan infinitas posibilidades de favorecer el aprendizaje, no solo a nivel disciplinar, sino también en actitudes y otros ámbitos de formación relevantes para el desarrollo de los jóvenes. Al final de cuentas, las TIC deben constituirse como uno más de los múltiples recursos que el docente debe aprovechar para crear espacios de interacción con sus alumnos donde se den situaciones que generen algún tipo de aprendizaje (Malaguzzi, 1980).

Según Moreira (1997), los ambientes de aprendizaje suelen ser muy variados, pero lo relevante es que sean diseñados de manera que sean significativos, por lo que no únicamente el aula puede entrar en esta clasificación, sino todo aquel lugar en el que el estudiante pueda aprender interactuando con sus pares, el ambiente y sus profesores. Por ende, las TIC son una herramienta que bien puede constituirse para generar nuevos ambientes de aprendizajes.

Por otro lado, la progresiva miniaturización de los componentes tecnológicos ha permitido que los ordenadores y dispositivos sean cada vez más portátiles, convirtiendo su uso en algo cotidiano por los jóvenes estudiantes y que puede convertirse en un factor claramente utilizado por los profesores, sin importar el área de formación a la que pertenezcan (Muñoz, 2010).

Es posible citar múltiples estudios que engloban el impacto del internet (Duart, 2009), los dispositivos móviles, las redes sociales (Rosa, 2016; Omart \& Navés, 2014) y otras tecnologías en el fenómeno educativo, pero al final de cuentas lo relevante es comprender que estos 
medios electrónicos deben de ser vistos como una oportunidad de versatilidad la educación y la manera en que los docentes pueden llegar a interactuar y aportar a la formación de sus alumnos.

Una tecnología que en los últimos años ha resultado novedosa en el ámbito educativo (aún más novedosa que lo mencionado anteriormente) es el uso de realidad aumentada, es decir, la superposición en una imagen real obtenida a través de una pantalla de imágenes, modelos 3D u otro tipo de informaciones generadas por ordenador (Prendes, 2015). Esta tecnología parece estarse usando para múltiples proyectos, pues permite la mezcla de la realidad física del usuario con elementos virtuales disponiendo así una realidad mixta en tiempo real (De Pedro, 2011).Como lo plantea Basogain, Olabe, Espinosa, Eouéche y Olabe (2007), la realidad aumentada no reemplaza el mundo real, sino al contrario, mantiene el mundo real que ve el usuario complementándolo con información virtual superpuesta a la real. Así, el usuario no pierde lo real, sino simplemente puede interactuar con la información virtual superpuesta.

Existen múltiples programas de realidad aumentada que se usan hoy en día en temas educativos, como por ejemplo Google Sky Map, FETCH! Lunch Rush, ZooBurst, AcrossAir, entre otros. Sin embargo, lo relevante no es únicamente usar estos programas que específicamente están dirigidos al entorno educativo, sino también, el poder aprovechar cualquier oportunidad por medio de las TIC, para que el alumno, a partir de una realidad extendida, pueda adquirir conocimientos. Esto es lo que se pretende con el presente artículo, el cual a partir de un juego de realidad aumentada, Pokémon Go, busca enmarcar una experiencia de aprendizaje con la que alumnos de pregrado de una universidad privada de Zapopan, México han conseguido desarrollar la subcompetencia ética de reconocimiento.

Aunque el objetivo primordial de este juego, que ha resultado ser una megatendencia entre los jóvenes durante el verano del 2016, no es específicamente la educación, sí consigue configurarse como un ambiente de aprendizaje, en donde el jugador se acerca a su realidad a partir de la realidad virtual, conjugándose múltiples posibilidades de reconocer necesidades o problemáticas de impacto social. Así, este texto busca arrojar nueva luz sobre el uso de TIC para la formación con sentido humano, específicamente al acercar al estudiante a una realidad que exige su atención. 


\section{EI reconocimiento como primer pasó para la empatía y la sensibilidad social}

Durante las últimas décadas, el tema del reconocimiento parece constituirse como un tópico central en los debates políticos, como consecuencia del claro aumento en los movimientos sociales alrededor del mundo. Taylor (2009) considera que esto se debe a un punto central; el que los individuos o los grupos deben ver sus diferencias, reconocerlas y aprender a respetarlas, sobre todo en el mundo globalizado en el que actualmente nos desarrollamos.

Es importante señalar que el reconocimiento permite construir un espíritu de solidaridad con los otros que nos rodean, dando pauta a una evolución moral de las sociedades enteras, en las que el yo y los otros se encuentran y dan pauta a un sentido comunitario de lo que es bueno y valioso. Para los eticistas, una persona solo puede acercarse a una vida correcta, cuando su manera de actuar es capaz de suscitar la estima en el seno de su grupo, es decir, cuando puede reconocer al otro y el otro lo reconoce a él (Canto-Sperber, 2001).

El reconocimiento, es una manera en la que el individuo cultiva la empatía ante los problemas de aquellos quienes lo rodean, ya que negarlos o no preocuparse por ellos, sería tan grave como no reconocer la vulnerabilidad moral que cada ser humano tiene en el transcurrir de su propia vida. El reconocimiento es el primer paso en el presupuesto esencial que todo individuo tiene de actuar ante los problemas de su sociedad, la cual le reclama un actuar proporcional a los problemas a los que se enfrenta. En palabras de David Noel Ramírez, es necesario que el individuo piense en todo momento en aquellos quienes viven situaciones difíciles, tanto económica como moralmente, pues toca a todos los demás, el liberarlos del sufrimiento (Ramirez, 2014).

Lamentablemente, en una sociedad como la contemporánea, en que las actividades diarias saturan los tiempos de los individuos, es cada vez más usual una indiferencia social ante la realidad (Red Distrital de Comunicación Interna -RDCI-, 2007). Por si esto fuera poco, en las últimas décadas el ser humano parece tener menos tolerancia ante aquello que le resulta desafiante, prefiriendo abrazarse a una cómoda indiferencia, que enfrentar situaciones que le abruman o le resultan disonantes (Gómez-Ramírez \& Reyes Cruz, 2008). El reconocimiento, al confirmar la autonomía moral del individuo, da pauta a una obligación recíproca de igualdad universal en la manera en que se trata a las demás personas, ya que todos los individuos tienen, unos respecto a otros, el deber de respetarse y tratarse como personas, con la misma facultad de discernimiento 
moral (Gil, 2015).

Ninguna institución educativa puede negar que la actividad profesional es un comportamiento social y que por ende, obliga a la interacción, la convivencia y el reconocimiento en sí y en los demás de la dignidad y derechos que promueven una relación de respeto, reciprocidad y justicia entre todos. Esto es lo que motiva a que se considere la importancia del reconocimiento como una parte fundamental de la formación con sentido humano, e incluso, como una parte del desarrollo de una competencia ética en los futuros profesionistas. Sin embargo, ¿cómo desarrollar el reconocimiento cuando el individuo no tiene una interacción real con la sociedad que lo rodea?, ¿cómo se espera despertar el interés en los problemas sociales, cuando la persona desconoce la existencia de los mismos?

El presente artículo, por medio de una experiencia educativa en alumnos de nivel universitario, busca proponer una forma en que los juegos de realidad aumentada pueden despertar un interés palpable en los jóvenes por su realidad, al conjugar la sociedad que los rodea con el ambiente virtual que la aplicación les presenta. De esta forma, se pueden generar propuesta de impacto social, como las que el siguiente caso de estudio plantea.

\section{El caso de estudio}

Pokémon Go es un videojuego de realidad aumentada basada en la geolocalización desarrollado por Niantic, Inc. y Nintendo. El juego consiste en buscar, capturar y luchar con los personajes de la saga Pokémon escondidos en ubicaciones del mundo real, lo que lleva consigo a que el jugador deba desplazarse físicamente por las ciudades para progresar (BBC, 2016). Se determinó que para julio del 2016 el número de usuarios en Estados Unidos superaba los 20 millones, y que a nivel mundial, había más de 200 millones de jugadores activos (Fernández J. , 2016). Esto es lo que lleva a que Pokémon Go se convierta en la aplicación ideal para despertar el interés en los jóvenes, por ser la megatendencia del 2016.

Para este estudio, se ha realizado una actividad colaborativa con dos grupos de estudiantes universitarios de una institución privada del municipio de Zapopan, México. En total, la actividad se dio con la participación de 57 individuos, durante las dos últimas semanas del mes de agosto del 2016, en donde, por medio de equipos de trabajo colaborativos, los estudiantes 
tuvieron que hacer frente al siguiente reto: Proponer una actividad en donde jugando Pokémon Go, se hicieran conscientes de una necesidad o problema social, buscando desarrollar alternativas para su solución o mejora.

Para conseguir este objetivo, se les dio la tarea de salir y relacionarse con su entorno, buscando que el deseo de jugar, trajera consigo la posibilidad de conocer la realidad que los rodea, tanto en los alrededores de su institución universitaria, como en sus trabajos y sus hogares. Habiendo planteado el reto, los estudiantes tuvieron cinco días para realizar sus propuestas, siendo las siguientes (Tabla 1: Reconocimiento de la realidad con Pokémon Go), las consideradas más interesantes, tanto por su viabilidad, como por su posible impacto:

Tabla 1: Reconocimiento de la realidad con Pokémon Go.

\begin{tabular}{|c|c|}
\hline Problema que impacta & Propuesta \\
\hline $\begin{array}{l}\text { Falta de conocimiento } \\
\text { y apoyo a asociaciones } \\
\text { civiles. }\end{array}$ & $\begin{array}{l}\text { Hacer que las asociaciones civiles sean pokeparadas, lo que haría } \\
\text { que más personas supieran donde se ubican. Además, se podría } \\
\text { generar una campaña donde un porcentaje de las donaciones } \\
\text { realizadas pudieran ser canjeables por créditos en la aplicación. }\end{array}$ \\
\hline $\begin{array}{l}\text { Toma de consciencia } \\
\text { de sitios vulnerables, } \\
\text { así como la activación } \\
\text { económica de zonas de } \\
\text { bajos recursos }\end{array}$ & $\begin{array}{l}\text { Hacer eventos en los que por medio de la aparición de pokemones } \\
\text { raros o de alto nivel de puntos de combate se busque incentivar } \\
\text { la asistencia de jugadores a zonas de bajos recursos, promoviendo } \\
\text { la toma de consciencia de esta realidad, así como la activación } \\
\text { económica, buscando que los vecinos ofrezcan servicios a los } \\
\text { visitantes. Esto puede también utilizarse para promover el } \\
\text { turismo en regiones poco visitadas o que requieran de apoyo } \\
\text { económico, como las zonas rurales o las comunidades indígenas. }\end{array}$ \\
\hline $\begin{array}{l}\text { Campañas urbanas de } \\
\text { limpieza }\end{array}$ & $\begin{array}{l}\text { Promover campañas de recolección de basura en algunas zonas } \\
\text { de la ciudad, como parques, jardines o plazas públicas por medio } \\
\text { un apoyo mutuo entre los jugadores y personal de limpieza del } \\
\text { municipio. El punto central sería el canje de pequeñas bolsas de } \\
\text { basura recolectadas en la zona a cambio de la posibilidad de } \\
\text { cargar batería para su dispositivo o bien, botellas de agua para } \\
\text { poder continuar su cacería. }\end{array}$ \\
\hline $\begin{array}{l}\text { Programas } \\
\text { activación física }\end{array}$ & $\begin{array}{l}\text { Generar que durante los fines de semana se den recorridos que } \\
\text { por un lado permita a jugadores el poder recolectar pokemones } \\
\text { atraídos por medio de cebos en diferentes pokeparadas, pero que }\end{array}$ \\
\hline
\end{tabular}




\begin{tabular}{|l|l|}
\hline & $\begin{array}{l}\text { por otro lado sean recorridos con módulos intermitentes que los } \\
\text { obliguen a caminar y con ello activarse físicamente. }\end{array}$ \\
\hline $\begin{array}{l}\text { Apoyo alimentario a } \\
\text { causas sociales }\end{array}$ & $\begin{array}{l}\text { Ubicar módulos que otorguen servicios a los jugadores en zonas } \\
\text { concurridas de actividad Pokémon, con el fin de intercambiar } \\
\text { víveres a cambio de batería y agua. Todo lo recolectado puede } \\
\text { ser donado a bancos de alimentos u otras causas sociales. }\end{array}$ \\
\hline
\end{tabular}

Fuente: Creación propia

Como es posible apreciar en la Tabla 1, los participantes no únicamente pudieron identificar algunos de los problemas que más aquejan a la sociedad contemporánea, como la pobreza, el hambre, los problemas sociales, las zonas vulnerables, la falta de actividad física o el problema medioambiental de las ciudades, sino que también consiguieron sensibilizarse sobre la necesidad de hacer algo en beneficio de la sociedad. Aunque el juego propuesto no tiene un objetivo social, esta actividad nos demuestra que el impacto depende en gran medida del uso que se da de las cosas y no siempre del fin original de las mismas.

\section{Referencias internacionales a este tipo de actividades}

No se pretende con este artículo hacer pensar que lo propuesto resulta ser original o único, ya que como parte de la investigación previa al reto, los estudiantes tuvieron que informarse sobre ejemplos semejantes que se han dado en otras partes del mundo. Algunos de estos casos son:

Tabla 2: Ejemplos del uso social de Pokémon Go

\begin{tabular}{|l|l|}
\hline Nombre & Actividad \\
\hline \#PokemonInSyria & $\begin{array}{l}\text { Difundir por redes sociales fotografías de niños sirios con carteles } \\
\text { con dibujos de Pokémon, con la intención de sensibilizar sobre el } \\
\text { impacto de la guerra en la niñez de dicha zona (Main, 2016). }\end{array}$ \\
\hline $\begin{array}{l}\text { Limpieza } \\
\text { Equipo }\end{array}$ & $\begin{array}{l}\text { Limpieza de los parques Cole en Nueva York y el Centro de } \\
\text { Wichita, Kansas por medio de eventos de recolección de basura } \\
\text { organizada para jugadores (Anderson, 2016). }\end{array}$ \\
\hline $\begin{array}{l}\text { Refugio Muncie } \\
\text { para perros sin } \\
\text { hogar }\end{array}$ & $\begin{array}{l}\text { El refugio para animales Muncie, en Indianápolis, propuso la renta } \\
\text { de los perros que tenía en adopción para que los jugadores pudieran } \\
\text { pasearlos mientras jugaban. El dinero, permitió continuar con las } \\
\text { labores del refugio, pero también generó que muchos de los }\end{array}$ \\
\hline
\end{tabular}




\begin{tabular}{|l|l|}
\hline & $\begin{array}{l}\text { visitantes terminaran adoptando a los perros dejando al refugio sin } \\
\text { animales en pocas semanas de iniciar su campaña (Chan, 2016). }\end{array}$ \\
\hline $\begin{array}{l}\text { Mejora de salud } \\
\text { mental }\end{array}$ & $\begin{array}{l}\text { El psicólogo John M. Grohol de PsychCentral ha puesto como parte } \\
\text { de la terapia de sus pacientes con depresión el jugar Pokémon Go, } \\
\text { ya que ha demostrado el uso de la aplicación resulta ser una } \\
\text { motivación para muchos de sus pacientes para levantarse y salir de } \\
\text { su casa, algo que es a menudo una lucha para las personas con } \\
\text { trastornos depresivos (Macdonald, 2016). }\end{array}$ \\
\hline
\end{tabular}

Fuente: Creación propia

Como estos, existen múltiples casos en los que el gran impacto de Pokémon Go ha sido utilizado con fines sociales, sin embargo, lo relevante de lo planteado por este artículo no son tanto las propuestas, sino más bien la manera de desarrollar habilidades de reconocimiento social en los jóvenes, lo cual, requiere de ambientes de aprendizaje muy variados, como el que se ha utilizado en este ejemplo.

\section{Discusión de resultados}

Hablar hoy en día de objetivos institucionales en las universidades, ya no únicamente abarcan el área disciplinar y técnica que se aporta a los estudiantes, sino también, la necesidad de formar un sentido humano y una sensibilidad moral hacia el entorno y los problemas que los rodean. Aunque se desarrollen currículas que incluyan el abordaje y reflexión sobre la realidad social, estas no siempre exponen al alumno a dicha realidad, lo que hace que las competencias no se desarrollen de manera plena. La intención de acercar al joven al espectro social, como se ha conseguido con la experiencia planteada, trae consigo la necesidad de que éste reconozca por sí mismo los retos que le esperan en un futuro, lo que le significa poder desarrollarse como un profesionista íntegro (Schön, 1992).

El desarrollo de la competencia ética, aunque sí se beneficia de la reflexión teórica, exige el acercamiento a la realidad, ya que, como lo señala Beuchamp (2003, pág. 5): Las teorías generales están mal preparadas, aunque más bien son incapaces para ser llevadas a la práctica, porque resuelven problemas filosóficos que están, en sí mismos, desvinculados de la realidad.

Reflexionar sobre el marco general de la moralidad y la ética, no es lo mismo que hacer una aproximación a los problemas morales o éticos que se dan en la sociedad. Esto puede 
apreciarse en la mayoría de los manuales de ética, mismos que aunque vienen acompañados de un consistente apartado teórico, terminan abordando las problemáticas a partir de una reflexión de casos concretos y perspectivas propias desarrolladas por el autor (Velásquez, 2006; Weiss 2009; Crane y Matten, 2009). Este panorama de formación ética en un sentido ampliado y práctico, permite que el estudiante perciba que lo aprendido no es algo ajeno a lo que vive cada día, sino más bien, algo que se apega a la realidad que enfrentarán como profesionistas. Por ello, cualquier herramienta que acerque al individuo a su entorno, y más en el desarrollo de una competencia, resulta tan relevante.

El profesor que pueda incluir en sus actividades este tipo de técnicas encontrará en el presente artículo una excelente manera de que la formación ética no parezca como algo alejado de lo que el alumno vive cada día, sino más bien, como un aspecto que automáticamente debe considerarse como parte del entorno al que pertenece y más cuando la intención sea contribuir a los problemas que enfrenta la sociedad. La propuesta que se planteó en el presente artículo busca responder a esta necesidad, al utilizar una herramienta novedosa dentro del salón de clases como un ambiente de aprendizaje alternativo a lo tradicional.

\section{Conclusiones}

Para Nieto (2000), la enseñanza universitaria debe verse vinculada con la sociedad y la cultura a partir del análisis de situaciones reales y por ende, debe estar en constante evolución si se pretende que la formación sea realmente integral. De esta forma es que la formación de competencias metadisciplinares a través de una aproximación a la realidad se constituye como un medio idóneo para conectar el aprendizaje disciplinar con el aprendizaje natural y social de los individuos.

Saber afrontar los problemas de la realidad, exige acercarse a la misma y conocerla, ya que solo de esta manera, se pueden plantear propuestas coherentes y que generen un verdadero impacto. Por ello, el formar futuros profesionistas debe traer consigo el desarrollar, de manera complementaria, una sensibilidad viva hacia los problemas de su sociedad, reconociendo el rol que se juega como agente decisor y la manera en que sus actos pueden generar una diferencia. 
La propuesta aquí presentada busca que el profesionista del mañana reconozca la competencia ética como una parte natural de su actuar, misma que le permita identificarse como un verdadero agente de cambio, responsable de sus actos y sujeto a las consecuencias de sus comportamientos. Aunque la actividad realizada se enfrenta a claros limitantes, al solo haberse aplicado en un número muy restringido de estudiantes, y enfocarse únicamente en una aplicación de realidad aumentada, no se puede negar su relevancia al arrojar nueva luz sobre el uso de este tipo de herramientas para el desarrollo de sub-competencias éticas. La ética debe vivirse para realmente desarrollarse como parte de la formación del estudiante universitario, ya que éste se desenvuelve en un entorno, y todo lo que se dé en el mismo es de su incumbencia, le es relevante y tiene la obligación de reconocer y formar parte. Al final de cuentas, la formación universitaria se constituye a partir de necesidades reales, lo que hace inevitable el que la currícula y la realidad desarrollen una constante interacción. 


\section{Referencias}

Anderson, A. (Julio de 2016). Pokémon Go players gather to clean up downtown Wichita. KSN.com. Recuperado el Septiemnre de 2016, de http://ksn.com/2016/07/22/pokemon-goplayers-gather-to-cleanup-downtown-wichita/

Basogain, X., Olabe, M., Espinosa, K., Rouéche, C., \& Olabe, J. C. (2007). Realidad Aumentada en la Educación: Una tecnología emergente. Educa Madrid 2007: 7ma. Conferencia Internacional de Educación y la Formación basada en las Tecnologías. Madrid.

BBC. (07 de Julio de 2016). ¿Qué es Pokemon Go? La Nación. Recuperado el Septiembre de 2016, de http://www.lanacion.com.ar/1916290-que-es-pokemon-go-el-juego-de-celular-quepone-a-la-gente-a-caminar-y-ya-causo-problemas-con-la-policia

Best, F. (02 de 2015). Education, Culture, Human Rights and International Understanding: The Promotion of Humanistic, Ethical and Cultural Values in Education. Obtenido de http://unesdoc.unesco.org/images/0009/000914/091422eb.pdf

Beuchamp, T. L. (2003). The Nature of Applied Ethics. En R. G. Frey , \& C. H. Wellman, $A$ Companion to Applied Ethics (págs. 1-16). Malden: Blackwell.

Canto-Sperber, M. (2001). Reconocimiento. En M. Canto-Sperber, Diccionario de ética y de filosofía moral. México: Fondo de Cultura Económica.

CEFyTIC. (2014). Recursos educativos digitales para la educación infantil. (C. E. TIC, Ed.) Zona Próxima, 1-21.

Chan, M. (Julio de 2016). This animal shelter wants Pokémon Go players to help walk its dogs. TIME. Recuperado el Septiembre de 2016, de http://time.com/4403865/pokemon-gomuncie-animal-shelter-dog-walk/

Crane, A., \& Matten, D. (2010). Business Ethics. Managing Corporate Citizenship and Sustainability in the Age or Globalization. Oxford: Oxford University Press.

De Pedro, J. (2011). Realidad Aumentada: un nuevo paradigma en la educaicón superior. En E. Campo, M. García, E. Meziat, \& L. Bengochea, Educación y Sociedad (págs. 300-307). Chile: Universidad La Serena.

Duart, J. (2009). Internet, redes sociales y educación. RUSC. Universities and Knowledge Society Journal, 6(1), 1-2.

Fernández, J. (16 de Julio de 2016). La fiebre Pokémon en cifras. EXPANSION. Recuperado el Septiembre de 2016, de http://www.expansion.com/economiadigital/companias/2016/07/16/57890adc22601db1308b45d9.html

Fernández, J. M., \& Velazco, N. (2003). La transversalidad curricular en el contexto de la enseñanza superior. Agenda Académica, 10(2), 61-69. 
Gil, J. (2015). El reconocimiento afectivo como contenido moral. Tópicos, Revista de Filosofia(49), 53-79.

Gómez-Ramírez, O., \& Reyes Cruz, L. (2008). Las jóvenes y el feminismo: ¿Indiferencia o compromiso? Revista Estudios Feministas, 16(2), 387-408.

Harvard. (2015). Derek Bok Center for Teaching and Learning. Obtenido de Harvard University. Faculty of Arts and Sciences: http://bokcenter.harvard.edu/

Hernández, L., \& Muñoz, L. (2012). Usos de las tecnologías de la información y la comunicación (TIC) en un proceso formal de enseñanza y aprendizaje en la educación básica. Zona Próxima(16), 2-13.

Iracheta, F. (2011). ¿Qué significa una formación ética? En-claves del pensamiento, 5(10).

Macdonald, F. (12 de Julio de 2016). Pokémon Go is reportedly helping people with their depression. Science Alert. Recuperado el Septiembre de 2016, de http://www.sciencealert.com/pokemon-go-is-reportedly-helping-people-with-theirdepression

Main, E. (22 de Julio de 2016). Cómo se está utilizando Pokémon Go para llamar la atención sobre la situación en Siria. $B B C$. Recuperado el Septiembre de 2016, de http://www.bbc.com/mundo/noticias-36866945

Malaguzzi, L. (1980). Pedagogía como arte: el niño sin el medio ambiente y sin cosas. Recuperado el Septiembre de 2016, de http://www.educadorasdeinfantil.es/?p=86

Moreira, M. A. (1997). Aprendizaje significativo: un concepto subyacente. En M. A. Moreira, M. C. Caballero, \& M. L. Rodríguez, Actas del Encuentro Internacional sobre el Aprendizaje Significativo (págs. 19-44). Burgos. Recuperado el Septiembre de 2016, de http://www.if.ufrgs.br/ Moreira/apsigsubesp.pdf

Muñoz, C. (2010). Dispositivos móviles en la educación médica. Teoría de la Educación. Educación y Cultura en la Sociedad de la Información, 11(2), 28-45.

Nieto, S. (2000). Discurso del profesorado universitario sobre la educación del aprendizaje de los alumnos como estrategia de innovación y cambio profesional. Revista de Educación(322), 305-324.

Omart, E., \& Navés, A. (enero-junio de 2014). El uso de redes sociales como soporte educativo. CPU-e, Revista de Investigación Educativa(18), 172-181.

Prendes, C. (2015). Realidad aumentada y educación: análisis de experiencias prácticas. Pixel-Bit. Revista de Medios y Educación(46), 187-203.

Ramirez, D. N. (2014). Hipoteca Social. México: McGraw Hill. 
RDCI. (2007). La comunicación en una ciudad sin indiferencia. Signo y Pensamiento, XXVI(51), 185-187.

Redón, S. (2007). Significados de la transversalidad en el currpiculum: Un estudio de caso. Revista Iberoamericana de Educación(43).

Rosa, M. (2016). Redes sociales y educación. Teoría de la Educación. Educación y CUltura en la Sociedad de la Información, 17(2), 15-20.

Ruiz, M. (2004). Las TIC, un reto para nuevos aprendizajes. Madrid: Marcea.

Safra, E. J. (2015). Edmond J. Safra Philanthropic Foundation. Obtenido de Center for Ethics, Harvard University: http://www.edmondjsafra.org/education/edmond-j-safra-center-forethics

Sancho, J. (2006). Tecnologías para transformar la educación. Madrid: Akal.

Schön, D. (1992). La formación de profesionales reflexivos. Hacia un nuevo diseño de la enseñanza y el aprendizaje en las profesiones. Barcelona: Paidós.

Taylor, C. (2009). El multiculturalismo y "la política del reconocimiento". Ciudad de México: Fondo de Cultura Económica.

Toffer, A. (1992). El "shock" del futuro. Barcelona: Plaza y Janés.

Velasquez, M. (2006). Ética en los negocios. Conceptos y casos. México: Pearson.

Weiss, J. W. (2009). Business Ethics. A Stakeholder \& Issues Management Approach. Mason: South-Western Cengage Learning. 